\title{
Comparison of three physical therapy interventions with an emphasis on the gait of individuals with Parkinson's disease
}

\author{
Comparação de três intervenções fisioterapêuticas com \\ ênfase na marcha de indivíduos com doença de Parkinson
}

\author{
Maria Eduarda Brandão Bueno, Ana Carolina dos Reis Andrello, Marcelle Brandão Terra, \\ Hevely Beatriz Celestino dos Santos, Júlia Marson Marquioli, Suhaila Mahmoud Smaili Santos*
}

Universidade Estadual de Londrina (UEL), Londrina, PR, Brazil

\begin{abstract}
Introduction: Gait impairments are one of the earliest signs reported by patients with Parkinson's disease (PD) and cause an increased number of falls and decreased quality of life among these patients. Objective: To compare the effectiveness of three physical therapy interventions using Rhythmic Cues (RC), Swiss Ball (SB) and Dual Task (DT), with an emphasis on gait treatment (step and stride length, duration and velocity), in individuals with PD. Methods: Quasi-randomized clinical trial addressing a sample composed of 45 individuals assigned to three groups. The individuals were assessed before and after the intervention protocol using the following: Modified Hoehn and Yahr Scale (HY), Unified Parkinson's Disease Rating Scale (UPDRS), Footprint analysis, Video Gait analysis, and Timed Up and Go Test (TUG). The groups were homogeneous concerning age, HY and UPDRS. Statistical analysis was performed using SPSS, version 20.0. Results: Statistically significant differences were found in all the variables analyzed in the RC and SB groups when compared in the pre- and post-intervention. With the exception of the TUG variable, the DT group presented statistically significant differences in all the remaining variables. Conclusion: The three interventions were effective for the outcomes under study, but the SB group presented the greatest magnitude of
\end{abstract}

\footnotetext{
* MEBB: Master student, e-mail: m.eduardafit@gmail.com ACRA: Master student, e-mail: ana_andrello@hotmail.com MBT: Master student, e-mail: celleterra@gmail.com HBCS: BS, e-mail: hevely_beatriz@yahoo.com.br JMM: MS, e-mail: juliamarquioli@gmail.com SMSS: PhD, e-mail: suhaila@uel.br
} 
change (effect size), while the RC group presented the greatest improvement in the temporal gait variables (duration and velocity) and TUG.

Keywords: Parkinson Disease. Physical Therapy Modalities. Gait.

\section{Resumo}

Introdução: As alterações na marcha são um dos sinais relatados mais precocemente pelos pacientes com doença de Parkinson (DP), ocasionando aumento do número de quedas e redução da qualidade de vida desses indivíduos. Objetivo: Comparar a efetividade de três intervenções fisioterapêuticas utilizando Pistas Rítmicas (RC), Bola Suíça (SB) e Dupla Tarefa (DT) com ênfase no tratamento da marcha (tamanho do passo, da passada, tempo e velocidade) em indivíduos com DP. Métodos: Foi realizado estudo do tipo ensaio clínico quase randomizado, com casuística composta por 45 indivíduos, divididos nos três grupos. Os indivíduos foram avaliados antes e após o protocolo de intervenção pelos seguintes instrumentos: Escala de Hoehn \& Yahr Modificada (HY), Escala Unificada de Avaliação da doença de Parkinson (UPDRS), Teste de impressão plantar (footprint), Análise de marcha porvídeo e Teste Timed Up and Go (TUG). Os grupos eram homogêneos em relação à idade, HYe UPDRS. Para análise estatística foi utilizado o programa SPSS 20. Resultados: Foram encontradas diferenças estatisticamente significantes em todas as variáveis analisadas no RC e SB quando comparados os valores pré e pós intervenção. No DT, as variáveis também apresentaram diferenças estatisticamente significantes, exceto o TUG.

Conclusão: As três intervenções foram efetivas para os desfechos estudados, porém o grupo que apresentou maior magnitude de mudança (tamanho do efeito) foi o $S B$, enquanto o RC apresentou maior porcentagem de melhora nas variáveis temporais da marcha (tempo e velocidade), bem como no TUG.

Palavras-chave: Doença de Parkinson. Modalidades de Fisioterapia. Marcha.

\section{Introduction}

Parkinson's disease (PD) is a progressive neurological disorder, the main symptoms of which include resting tremor, bradykinesia, plastic hypertonia and postural instability. In the more advanced stages of the disease, in which axial motor symptoms are evidenced, changes in posture and gait may occur. These are signs that are more frequently reported by patients and are characterized by disordered short steps that contribute to postural instability, narrower walking base, shorter stride length, difficulty in initiating and finalizing gait, and in overcoming obstacles and changing direction, which may freeze gait, cause festination, falls, and decrease the quality of life of these individuals (1-3).

In addition to motor symptoms, patients may develop non-motor symptoms, such as depression, autonomic dysfunction, sleep disorders, cardiorespiratory disorders, and mainly cognitive disorders $(4,5)$, that hinder one's ability to deal with multiple simultaneous tasks, revealing the insufficiency of compensatory motor mechanisms (6).
Drug therapy is effective in preserving function, mobility and quality of life among PD patients (7), but a combination of pharmacotherapy and rehabilitation is the ideal strategy to manage symptoms (8). Gait deficits and postural instability only partially respond to the drug therapies and these symptoms increasingly impact the lives of patients (9). Because physical therapy is among the main approaches to the treatment of PD, there is a constant search for better physical therapy intervention programs that would enable improved management of signs and symptoms.

In this sense, the Bobath concept can be used to treat individuals with functional, mobility and/or postural disorders accruing from lesions in the central nervous system (10). This treatment is based on functional problem-solving through sensory-motor recovery and is characterized by a particular way to observing, analyzing and interpreting the patient's motor performance in a given task (11). Three physical therapy interventions were selected for this study, namely: Rhythmic Cues, Swiss Ball and Dual Task. 
These tasks were required of three different groups based on the principles of the Bobath concept.

The first treatment proposed was Rhythmic Cues, as auditory and visual rhythmic cues are effective in improving the gait of individuals with PD; these improve step preparation (12) due to the greater attention demanded during the task (13). Studies reveal that rhythmic cues help PD patients to replace typical automatic gait control with a more conscious and perhaps, more discreet movement, making walking easier. In this case, increased cortical activity observed during directed movement would compensate for striatal dysfunction (14). The use of this technique is associated with altered patterns of neural activity, more specifically, with improved activity in the lateral premotor cortex (15).

The second strategy chosen was Swiss Ball because it is a tool that enables analyzing, assessing, and treating neurological patients, as it requires vestibular and proprioceptive feedback to obtain appropriate adaptive responses (16). Few studies, however, use this resource to analyze its effectiveness in affecting gait variables of individuals with PD.

The third strategy was treatment including dual tasks as they are a prerequisite in the functional performance of various activities of daily living (17). Patients with PD present both motor and cognitive deficits when performing a dual task (18), and when the dual task is performed simultaneously with walking, they present worsened motor performance, with a slower gait in which the phase of dual support is longer with a lower cadence, when compared to healthy people. This practice, however, offers the possibility of improving performance (19).

Therefore, this study's objective was to compare the effectiveness of three physical therapy interventions using Rhythmic Cues (RC), Swiss Ball (SB) and Dual Task (DT) with exercises based on the Bobath concept, focusing on treating the gait (step length, stride length, velocity, and duration) of individuals with PD.

\section{Methods}

A quasi-randomized clinical trial was conducted with samples composed of the consecutive entry of patients, developed in the physical therapy outpatient clinic at the State University of Londrina in association with the Centro de Atendimento Social
Ágape [Agape Social Care Center] at the Parish of Nossa Senhora das Graças in Londrina, PR, Brazil.

The sample was composed of 45 individuals referred by the neurological outpatient service of the Hospital das Clinicas, State University of Londrina. Participants were selected based on a statistical archive and medical records service according to the following criteria: individuals with PD determined by medical diagnosis based on the London Brain Bank's criteria (20): older than 50 years of age; with the PD between stages 1.5 and 3 as determined by the modified Hoehn and Yahr Scale.

Individuals presenting other types of Parkinsonism or other associated neurological diseases, vestibular, cardiovascular, musculoskeletal, cognitive or comprehension disorders, visual or auditory impairment that could affect motor performance, or individuals using walking orthosis or assistive devices, or under treatment other than drug therapy, were excluded. Individuals who missed therapy sessions more than three times or whose medication changed over the course of the study were considered losses.

Fifteen individuals were initially recruited for the Rhythmic Cues (RC) group. They were assessed and then received 24 physical therapy sessions (three months of intervention - Stage 1). The Swiss Ball (SB) group was composed of another 15 individuals, who were assessed and then received 24 sessions (three months of intervention - Stage 2) and finally, 15 individuals were assessed and recruited for the Dual Task (DT) group, following the same procedures as with the others (Stage 3).

All participants voluntarily consented to participate in the study and signed free and informed consent forms. The study was approved by the Institutional Review Board at the State University of Londrina, University Hospital (Opinion report No. 066/2011). The patients involved in the study were assessed by physical therapists, always by the same evaluator before and after the intervention protocol, using the following instruments: 1) Modified Hoehn \& Yahr Scale (HY - Degree of Disability Scale): is used to assess individuals' impairment and stage of the disease. This scale is able to rapidly and easily determine a patient's general condition. Its modified version comprises seven stages of classification to assess the severity of PD and basically includes global measures of signs and symptoms that permit classifying individuals regarding their level of impairment. Individuals classified in stages 1 to 3 
present mild to moderate impairment while stages 4 and 5 present severe impairment (21). 2) Unified Scale of Parkinson's Disease Rating Scale: assesses self-reported and clinically observed signs and symptoms of the disease. It is composed of 42 items divided into four domains: mental activity, behavior and mood; activities of daily living (ADLs); motor exploration and drug therapy complications. Scores in each item range from 0 to 4 ; the higher the score the greater the impairment caused by the disease. Only two domains were used in this study: II - activities of daily living (score from 0 to 52 points) and III - motor exam (score from 0 to 108 points), totaling 27 items (22). 3) Footprint analysis: assesses the linear variables of gait (walking base, step length and stride length). Each individual dips their feet in water-soluble paint and is encouraged to walk a distance of 5 meters on a paper walkway (23). 4) Video gait analysis: assesses temporal gait variables (duration for the distance walked and gait velocity). The patient walks a 12-meter track and is instructed to keep a normal and comfortable pace. The analysis considers the track's 10 central meters so that the first and last meters are disregarded as they are considered acceleration and deceleration. The number of steps is counted and timed in seconds. Velocity is calculated by dividing space by time. 5) Timed Up and Go Test (TUG): assesses the patient's agility in walking a short distance with changes of direction. It is a simple instrument that consists of analyzing the time spent by an individual to get up from a chair without arms, walk a 3-meter distance, turn and return to the chair. Longer times represent a greater risk of falls (24).

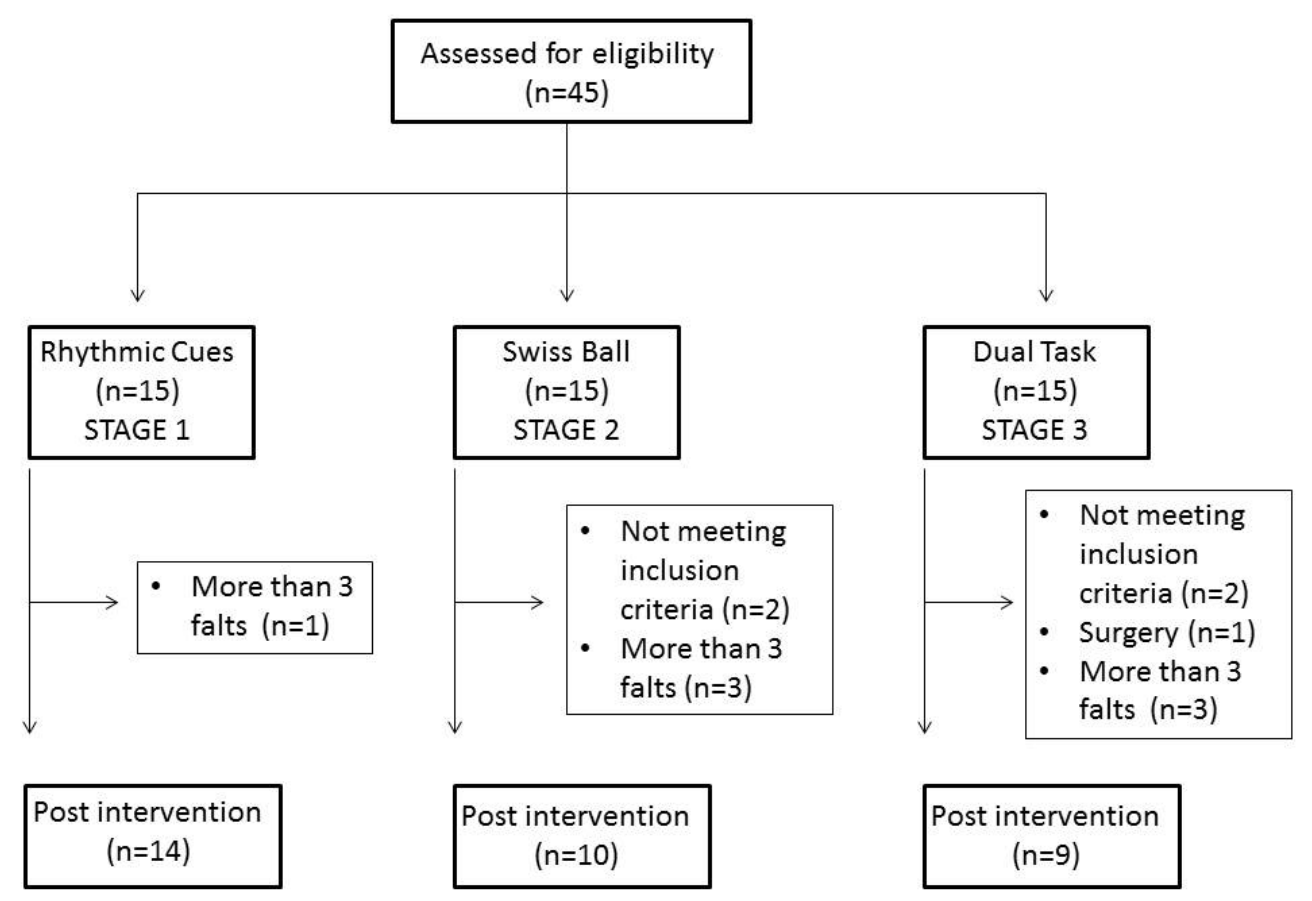

Figure 1 - Flowchart.

After each group was assessed according to the assessment protocol, the individuals received the therapeutic intervention proposed for each group and, at the end of their respective treatments, patients were reassessed. The same physical therapist implemented the treatment for each of the three groups and was blind regarding assessments and reassessments. The intervention was implemented in-group and each participant was monitored by a trained physical therapist. All the exercises were based on the Bobath concept, associated either with Rhythmic Cues, Swiss Ball or Dual Task, as follows:

\section{Rhythmic Cues Training}

This intervention protocol was divided into four phases, each composed of six sessions (totaling 24 
sessions), which developed with increasing complexity with two weekly sessions with a duration of 60 minutes each. Each session included three walking circuits using the following therapeutic devices: small ball, malleable stick, cones, hula-hoops, colored obstacles, horizontal ladders, step platform and chairs. The participants were supposed to perform the following tasks: walk according to the rhythm dictated by the physical therapist; catch and toss a ball; walk according to horizontal marks on the floor; walk up and down the step platform; and walk bypassing or circumventing obstacles. The patients were always monitored by a physical therapist during the visual and auditory rhythmic cues interventions. This therapist used verbal commands to guide them on the circuits as well as establish them the rhythm they were supposed to follow to perform the tasks.

\section{Swiss ball Training}

The training protocol consisted of 26 exercises; all included a Swiss ball as a therapeutic resource. There were two weekly sessions with a duration of 60 minutes, totaling 24 sessions. The exercises progressed with increasing complexity. The exercises started in the supine position and mobility exercises of lower and upper limbs were performed, along with coordinated exercises of lower and upper limbs.

A more complex exercise included kneeling and semi-kneeling positions that emphasized movements that stretched the patient's stability limitations, by oscillating the center of gravity, including trunk rotations, and position changes. Additionally, exercises that included sitting on the ball were implemented to improve strategies to maintain balance associated with changes in the center of gravity, rotational movements, and changing the walking base. At the end of the protocol, gait exercises were performed with the Swiss ball such as holding the ball and hitting it on the floor alternating with the upper limb, then hit the ball, and the lower limb, then take the steps.

\section{Dual Task Training}

This protocol was divided into three phases, each composed of eight sessions (totaling 24 sessions), with two weekly sessions with a duration of
60 minutes. This intervention also gradually became more complex. The materials used included: chair, plastic soccer ball, cones, colored boxes, hula-hoops, malleable stick, horizontal ladder, and poly half cones, in addition to posters with pictures, words or arithmetic operations for the dual tasks.

Progressive exercises were performed in the sitting and standing positions, progressing from simple to dual tasks such as rotating the trunk to both sides and subtracting 10 from 100 (by 10s), changing sitting position to standing position in the Romberg position while simultaneously naming figures positioned ahead, walking on a 12-meter circuit at maximum velocity while simultaneously naming types of sports during the entire track, among others.

\section{Statistical analysis}

In the descriptive analysis, data were presented according to the distribution of normality according to the Shapiro-Wilk test (mean, standard deviation or median and interquartile interval). The paired t-test was used to compare between intra-groups pre- and post-assessments. As a complement to statistical significance, effect size measurements (Cohen's d) were used to represent the magnitude of the results obtained in the procedures assessing the groups (25). To make inter-groups comparison, a variance analysis was performed using the Tukey posttest. Additionally, to verify the percentage of improvement, we divided the difference between the final-initial means $(\Delta)$ by the pre-intervention value. The statistical analysis was performed in the SPSS program, version 20 , with significance value established at $5 \%$.

\section{Results}

a) Intra-group results:

The participants' characteristics at the beginning of the study are presented in Table 1. The groups were homogenous in regard to age and clinical staging of the disease.

Table 2 presents the scores obtained by the three groups concerning Timed Up and Go, step and stride length, duration of distance walked, and gait velocity. Statistically significant differences were found in all the variables under study for the three treatment 
groups when comparing pre- and post-intervention values, except for the DT group in regard to the TUG variable $(\mathrm{p}=0.17)$.

In regard to the effect of treatment, the effect size of all the groups was considered large $(d>0.80)$, except for the variable TUG in the SB and DT groups, though the group that presented the highest magnitude of change, according to Cohen's $d$ test, was the SB group when comparing pre- and post-intervention, with the exception of the variable TUG.

Note that for the temporal gait variables such as TUG, duration of distance walked, and gait velocity, the RC group experienced a more expressive improvement while the SB group presented the highest percentage of change in regard to the variables that involve step and stride length.

Table 1 - Characteristics of participants

\begin{tabular}{lcccc}
\hline & $\begin{array}{c}\text { Rhythmic Cues } \\
(\mathbf{n}=\mathbf{1 4})\end{array}$ & $\begin{array}{c}\text { Swiss Ball } \\
(\mathbf{n}=\mathbf{1 0})\end{array}$ & $\begin{array}{c}\text { Double Task } \\
(\mathbf{n}=\mathbf{9})\end{array}$ & $\mathbf{P}$ \\
\hline Age (years) & $69.92 \pm 6.31$ & $70.90 \pm 7.65$ & $72.77 \pm 6.13$ & 0.59 \\
HY & $2.17 \pm 0.54$ & $1.85 \pm 0.33$ & $2.38 \pm 0.48$ & 0.56 \\
UPDRS (motor) & $28.35 \pm 10.03$ & $25.30 \pm 10.77$ & $20.11 \pm 10.04$ & 0.61 \\
UPDRS (ADL) & $11.92 \pm 6.25$ & $9.70 \pm 4.05$ & $10.00 \pm 5.09$ & 0.17 \\
UPDRS (total) & $40.28 \pm 15.06$ & $35.00 \pm 13.72$ & $30.11 \pm 13.47$ & 0.24 \\
\hline
\end{tabular}

Note: $\mathrm{HY}=$ Modified Hoehn \& Yarh Scale; UPDRS = Unified Scale of Parkinson's Disease Rating Scale; ADL = Activities of Daily Living.

Table 2 - Intra-group results

\begin{tabular}{lcccccc}
\hline VARIÁBLES & PRE & POST & $\Delta$ & d & P & $\%$ \\
\hline Rhythmic Cues & & & & & & \\
$\quad$ TUG (s) & $6.87 \pm 1.08$ & $4.95 \pm 1.03$ & $-1.91 \pm 0.61$ & -1.77 & 0.000 & 27.8 \\
$\quad$ Step lenght (cm) & $56.07 \pm 9.36$ & $66.35 \pm 9.55$ & $10.27 \pm 7.01$ & 1.10 & 0.000 & 18.3 \\
Stride lenght (cm) & $113.55 \pm 17.29$ & $131.10 \pm 18.27$ & $17.55 \pm 11.66$ & 1.02 & 0.000 & 15.4 \\
$\quad$ Duration (s) & $9.21 \pm 2.45$ & $5.35 \pm 1.59$ & $-3.86 \pm 2.18$ & -1.58 & 0.000 & 41.9 \\
$\quad$ Gait velocity (m/s) & $1.17 \pm 0.44$ & $2.02 \pm 0.59$ & $0.85 \pm 0.67$ & 1.93 & 0.000 & 72.6 \\
Swiss Ball & & & & & & \\
TUG (s) & $5.20 \pm 0.75$ & $4.68 \pm 0.38$ & $-0.58 \pm 0.61$ & -0.77 & 0.014 & 11.1 \\
Step lenght (cm) & $57.80 \pm 5.66$ & $71.00 \pm 11.40$ & $13.20 \pm 8.59$ & 2.33 & 0.001 & 22.8 \\
Stride lenght (cm) & $114.00 \pm 11.26$ & $142.11 \pm 18.88$ & $28.07 \pm 13.95$ & 2.49 & 0.000 & 24.6 \\
$\quad$ Duration (s) & $8.20 \pm 0.76$ & $5.89 \pm 0.62$ & $-2.28 \pm 1.02$ & -3.00 & 0.000 & 27.8 \\
Gait velocity (m/s) & $1.21 \pm 0.12$ & $1.69 \pm 0.19$ & $0.48 \pm 0.23$ & 4.00 & 0.000 & 39.6 \\
Dual task & & & & & & \\
$\quad$ TUG (s) & $6.52 \pm 0.82$ & $6.17 \pm 0.68$ & $-0.34 \pm 0.69$ & -0.41 & 0.17 & 5.2 \\
$\quad$ Step lenght (cm) & $57.26 \pm 7.62$ & $68.50 \pm 10.35$ & $11.23 \pm 7.95$ & 1.47 & 0.003 & 19.6 \\
Stride lenght (cm) & $115.73 \pm 12.39$ & $135.72 \pm 18.24$ & $19.98 \pm 13.41$ & 1.61 & 0.002 & 17.2 \\
$\quad$ Duration (s) & $7.63 \pm 0.58$ & $6.00 \pm 0.61$ & $-1.56 \pm 0.60$ & -2.69 & 0.000 & 20.4 \\
Gait velocity (m/s) & $1.31 \pm 0.09$ & $1.66 \pm 0.18$ & $0.34 \pm 0.16$ & 3.78 & 0.000 & 25.9 \\
\hline
\end{tabular}

Note: $\Delta=$ difference between the final-initial means; $\mathrm{d}=$ Cohen's d (effect size); \% (percentage of improvement) $=\Delta$ divided by the preintervention value; TUG $=$ Timed Up and Go test. 
b) Inter-groups results

To make inter-groups comparisons, we verified the difference between the groups' means and the statistically significant difference for the variables TUG, duration of distance walked, and gait velocity. The RC group presented superior performance compared to that of the SB and DT groups for the variables TUG and duration of distance walked. Differences in regard to the variable gait velocity were found only between the RC and DT groups. Hence, the group that stands out regarding the variables that relate to gait velocity was the RC group. These data are presented in Figure 2.

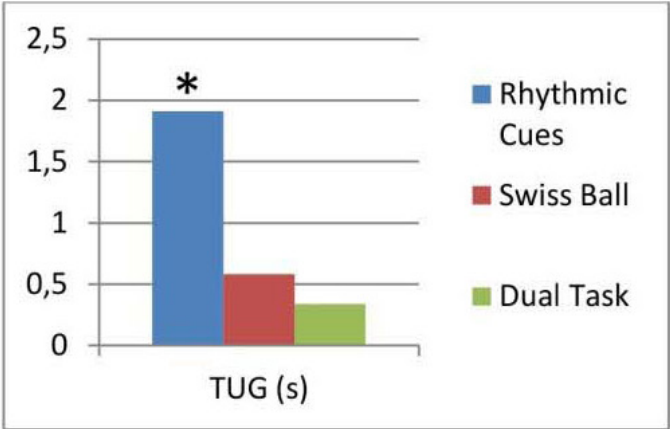

C

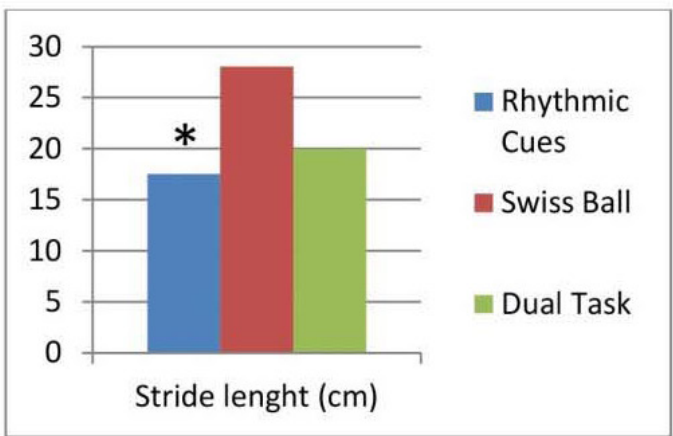

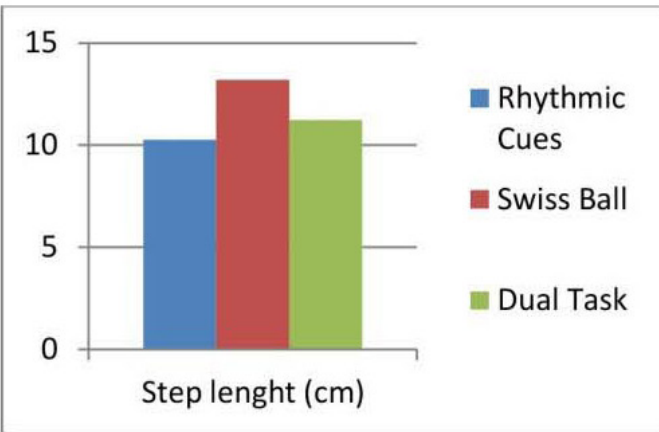

D

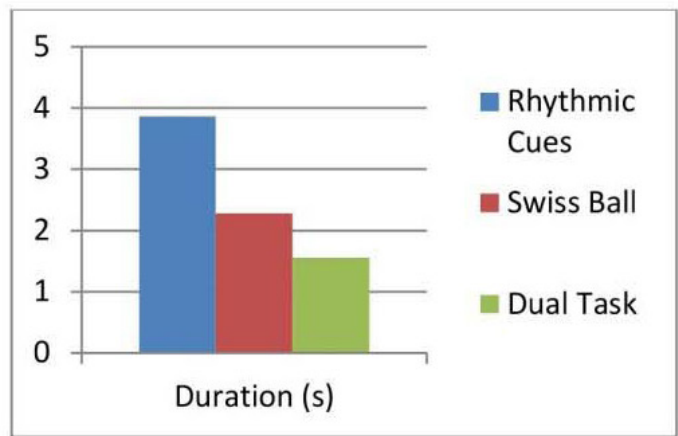

E

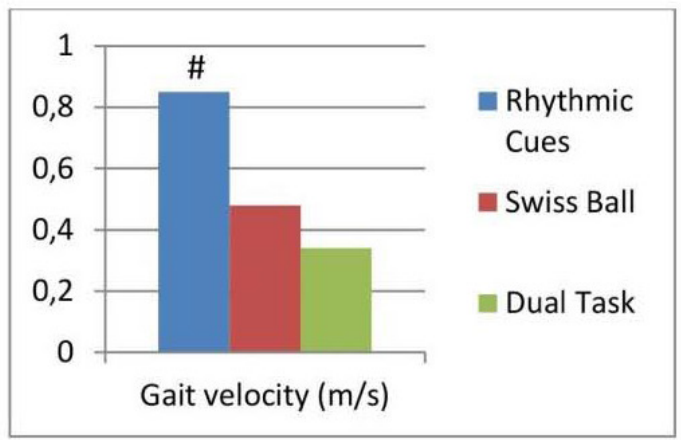

Figure 2 - Inter-group results.

Note: TUG = Timed Up and Go test; "Statistically significant differences between Rhythmic Cues, Swiss Ball and Dual Task groups; \#Statistically significant differences between Rhythmic Cues and Dual Task groups. 


\section{Discussion}

The inclusion of multidisciplinary rehabilitation in the drug and surgical treatment of PD has increased recently. Physical therapy for PD focuses on the posture, transfers, the function of the upper limbs, balance, gait, and physical capacity. The strategies used in the interventions aimed to keep or improve the individuals' independence, safety, and quality of life (26). There is well-established evidence in the literature showing that therapeutic exercise is able to delay the emergence of functional impairment in PD and a growing number of randomized clinical trials report that various types of exercises improve some aspects of balance and gait $(27,28)$.

There is, however, a difficulty in determining whether a specific type of exercise program is more efficacious than another because studies use different ways to measure results. Additionally, studies almost always compare an intervention protocol with a placebo intervention or no intervention at all (29). Due to ethical considerations and the fact it is a progressive neurodegenerative disease, we opted not to establish a control group since the assignment of patients to this group would harm their clinical progress, considering that the benefits of physical therapy for PD patients are well-established in the literature. Therefore, this study compared three physical therapy interventions to establish which would be the best approach to treat the gait of individuals with PD.

This study presents statistically significant improvement for almost all variables in all the groups, confirming that physical therapy is effective to manage PD. When the effect size of interventions was analyzed (which takes into account the standard deviation of the pre-intervention score), the SB group presented the highest magnitude of change. In the inter-groups comparison, the group that presented the highest percentage of improvement in comparison to the remaining groups was the RC group.

In regard to the effects of the Swiss ball, there are few studies in the literature that specifically use this resource to treat the gait of individuals with PD. Randomized clinical trials addressing PD $(30,31)$, with experimental and control groups, which analyzed balance and gait variables and used the Swiss ball as a therapeutic tool during treatment, showed significant improvement in variables such as TUG, balance, and gait velocity. These results are in agreement with this study's findings as all the variables related to gait showed improvement.

In regard to the rhythmic cues, a group of researchers (32) conducted a study to verify whether the therapy with cues is effective in improving gait and lessening the severity of motor symptoms in individuals with PD. Auditory cues were performed using a metronome, which corrected the width and frequency of steps through the beats of the gaits during the performance of various tasks and everyday situations. Statistically significant improvement was observed in regard to step length and gait velocity, variables that also improved in this study among individuals guided by rhythmic cues, used as a therapeutic resource.

In regard to the dual task treatment, Fok and collaborators (33) assessed the effects of dividing attention between walking and the performance of a secondary cognitive task in people with mild to moderate PD. The intervention group received a 30 -minute training period divided between taking big steps and performing subtractions (by 3s) while the control group did not receive any treatment. Stride length, gait velocity and number of accurate enumeration were verified at the beginning, immediately after and 30 minutes after training that included simple tasks (walking only or subtracting only) and dual task (walking while subtracting). Similar to this study, statistically significant differences were found in regard to stride length and gait velocity immediately after training.

In the same line of research, Brauer and Morris (34) conducted a study to assess 20 individuals with mild to moderate PD on both simple and dual tasks, before and after a 20-minute dual-task training session focused on improving stride length that was associated with additional memory tasks and progressive and regressive counting with verbal responses. Similar to this study, the participants presented shorter steps during gait performed with additional tasks prior to treatment; however, both stride length and gait velocity showed statistically significant improvement after the dual-task training. The authors revealed that a single training session is effective for these outcomes; however, a larger number of sessions, as we used in this study, may provide greater stimulus to neuroplasticity as recent evidence shows that exercises can induce a positive regulation of neurogenesis and reprogram synaptic neurotransmission (35). For this to occur, studies 
suggest longer interventions promoting continuous exercises to avoid inactivity (36).

One study conducted by Rochester and collaborators (37) associated rhythmic cues with dual tasks to test the effects of training on motor learning among individuals with PD, establishing acquisition (simple task), automaticity (dual task), and retention of simple and dual tasks (follow-up). Training, including gait with cues, was implemented among 153 individuals with PD over the course of three weeks and changes in gait performance with three external rhythmic cues (auditory, visual and somatosensory) was measured during simple and dual tasks after training and during a six-week follow-up. Gait velocity, frequency, and stride length were recorded using an accelerometer. Similar to this study, gait velocity and stride length improved significantly in the three types of cues after training during simple and dual tasks and such an effect remained after six weeks.

In summary, it is clear that the three groups experienced significant improvement (significant effect size) in the variables under analysis after different protocols were implemented. The SB group presented the greatest magnitude of change between pre- and post-intervention; however, comparison among groups revealed that the RC group's performance was superior to that of the SB and DT groups in regard to the gait temporal variables.

A potential explanation for this fact is that sensory resources (rhythmic and auditory cues) were used along with resources that promote instability (ball), challenging and requiring more mechanisms to recover balance, fine postural adjustments and the use of better strategies to keep the body stable. Additionally, the activities also encouraged changes of position and weight transfers, which surely improved the patients' perceptions regarding their stability limitations and gravity center adjustments. Possibly, together, these improved abilities positively impacted the individuals' gait pattern, as these are prerequisites for a dynamic and functional gait.

This study's limitations include lack of randomization, and for this reason, allocation bias may have occurred. Follow-up studies are needed to verify and reinforce the effects of the protocols in the long term, as well as the benefits experienced by patients with PD, in order to produce greater levels of scientific evidence.

\section{Conclusion}

The three interventions were effective for the outcomes under study but the group that presented the greatest magnitude of change when the effect size is estimated for the outcomes that included gait (step length, stride length, duration and velocity) in this population was the SB group. The RC group, however, presented the greatest improvement in the gait temporal variables (TUG, duration and velocity) compared to the SB and DT groups.

\section{References}

1. Frazitta G, Bertotti G, Uccellini D, Boveri N, Rovescala R, Pezzoli G, et al. Short- and long-term efficacy of intensive rehabilitation treatment on balance and gait in parkinsonian patients: a preliminary study with a 1-year followup. Parkinsons Dis. 2013;2013:583278.

2. Nemanich ST, Duncan RP, Dibble LE, Cavanaugh JT, Ellis TD, Ford MP, et al. Predictors of gait velocitys and the relationship of gait velocitys to falls in men and women with Parkinson disease. Parkinsons Dis. 2013;2013:141720.

3. Klucken J, Barth J, Kugler P, Schlachetzki J, Henze T, Marxreiter F, et al. Unbiased and mobile gait analysis detects motor impairment in Parkinson's disease. PLoS ONE. 2013;8(2):e56956.

4. Melo LM, Barbosa ER, Caramelli P. Declínio cognitivo e demência associados à doença de Parkinson: características clínicas e tratamento. Rev Psiquiatr Clín. 2007;34(4):176-83.

5. Trenkwalder, Kohnen R, Högl B, Metta V, Sixel-Döring F, Frauscher B, et al. Parkinson's disease sleep scale validation of the revised version PDSS-2. Mov Disord. 2011;26(4):644-52.

6. Baggio JAO, Curtarelli MB, Rodrigues GR, Tumas V. Validation of the brazilian version of the clinical gait and balance scale and comparison with the berg balance scale. Arq Neuropsiquiatr. 2013;71(9A):621-6.

7. Spaulding SJ, Barber B, Colby M, Cormack B, Mick T, Jenkins ME. Cueing and gait improvement among people with Parkinson's disease: a meta-analysis. Arch Phys Med Rehabil. 2013;94(3):562-70. 
8. Santos VV, Araújo MA, Nascimento OJM, Guimarães FS, Orsini M, Freitas MRG. Effects of a physical therapy home-based exercise program for Parkinson's disease. Fisioter Mov. 2012;25(4):709-15.

9. Grabli D, Karachi C, Welter ML, Lau B, Hirsch EC, Vidailhet $\mathrm{M}$, et al. Normal and pathological gait: what we learn from Parkinson's disease. J Neurol Neurosurg Psychiatry. 2012;83(10):979-85.

10. Graham JV, Eustace C, Brock K, Swain E, Irwin-Carruthers S. The Bobath concept in contemporary clinical practice. Top Stroke Rehabil. 2009;16(1):57-68.

11. Pagnussat AS, Simon AS, Santos CG, Postal M, Manacero S, Ramos RR. Atividade eletromiográfica dos extensores de tronco durante manuseio pelo Método Neuroevolutivo Bobath. Fisioter Mov. 2013;26(4):855-62.

12. Dias NP, Fraga DA, Cacho EWA, Oberg TD. Treino de marcha com pistas visuais no paciente com doença de Parkinson. Fisioter Mov. 2005;18(4):43-51.

13. El-Tamawy MS, Darwish MH, Khallaf ME. Effects of augmented proprioceptive cues on the parameters of gait on individuals with Parkinson's disease. Ann Indian Acad Neurol. 2012;15(4):267-72.

14. Vitorio R, Lirani-Silva E, Pieruccini-Faria F, Moraes R, Gobbi LTB, Almeida QJ. Visual cues and gait improvement in Parkinson's disease: which piece of information is really important? Neuroscience. 2014;277:273-80.

15. Young WR, Rodger MW, Craig CM. Auditory observation of stepping actions can cue both spatial and temporal components of gait in Parkinson's disease patients. Neuropsychologia. 2014;57:140-53.

16. Carrière B. Bola suíça: teoria, exercícios básicos e aplicação clínica. São Paulo: Manole; 1999.

17. Floriano EN, Alves JF, Almeida IA, Souza RB, Christofoletti G, Santos SMS. Dual task performance: a comparison between healthy elderly individuals and those with Parkinson's disease. Fisioter Mov. 2015;28(2):251-8.

18. Yogev-Seligmann G, Giladi N, Brozgol M, Hausdorff JM. A training program to improve gait while dual tasking in patients with Parkinson's disease: a pilot study. Arch Phys Med Rehabil. 2012;93(1):176-81.

19. Marinho MS, Chaves PM, Tabaral TO. Dupla-tarefa na doença de Parkinson: uma revisão sistemática de ensaios clínicos aleatorizados. Rev Bras Geriatr Gerontol. 2014;17(1):191-9.
20. Hughes AJ, Daniel SE, Kilford L, Lees AJ. Accuracy of clinical diagnosis of idiopathic Parkinson's disease. A clinico-pathological study of 100 cases. J Neurol Neurosurg Psychiatry. 1992;55(3):181-4.

21. Hoehn MM, Yahr MD. Parkinsonism: onset, progression and mortality. Neurology. 1967;17(5):427-42.

22. Fahn S, Marsden CD, Calne D, Goldstein M, editors. Recent Developments in Parkinson's Disease. Florham Park, N.J.: Macmillan Healthcare Information; 1987. p.153-63.

23. Romero VM. Análise das variáveis de distância da marcha de pacientes com acidente vascular encefálico. Consc Saúde. 2008;7(3):324-9.

24. Podsiadlo D, Richardson S. The timed up go: a test of basic functional mobility for elderly persons. J Am Geriatr Soc. 1991;39(2):142-8.

25. Cohen J. Statistical Power Analysis for the Behavioral Sciences. Lawrence Earlbaum Associates: Hillsdale; 1988.

26. Tomlinson CL, Patel S, Meek C, Herd CP, Clarke $\mathrm{CE}$, Stowe R, et al. Physiotherapy intervention in Parkinson's disease: systematic review and metaanalysis. BMJ. 2012;345:e5004.

27. Ebersbach G, Ebersbach A, Edler D, Kaufhold O, Kusch M, Kupsch A, et al. Comparing exercise in Parkinson's disease - the Berlin LSVT BIG study. Mov Disord. 2010;25(12):1902-8.

28. Earhart GM, Williams AJ. Treadmill training for individuals with Parkinson disease. Phys Ther. 2012;92(7):893-7.

29. King LA, Salarian A, Mancini M, Priest KC, Nutt J, Serdar A, et al. Exploring Outcome Measures for Exercise Intervention in People with Parkinson's Disease. Parkinsons Dis. 2013;2013:572134.

30. Christofoletti G, Freitas RT, Cândido ER, Cardoso CS. Effectiveness of a physical therapy treatment on static and dynamic balance of subjects with Parkinson's disease. Fisioter Pesqui. 2010;17(3):259-63.

31. Martínez-Amat A, Hita-Contreras F, Lomas-Vega R, Caballero-Martínez I, Alvarez PJ, Martínez-López E. Effects of 12-week proprioception training program on postural stability, gait, and balance in older adults: a controlled clinical trial. J Strength Cond Res. 2013;27(8):2180-8. 
32. Rochester L, Rafferty D, Dotchin C, Msuya O, Minde V, Walker RW. The effect of cueing therapy on single and dual-task gait in a drug naive population of people with Parkinson's disease in northern Tanzania. Mov Disord. 2010;25(7):906-11.

33. Fok P, Farrell M, McMeeken J. The effect of dividing attention between walking and auxiliary tasks in people with Parkinson's disease. Hum Mov Sci. 2012; 31(1):236-46.

34. Brauer SG, Morris ME. Can people with Parkinson's disease improve dual tasking when walking? Gait Posture. 2010; 31(2):229-33.

35. Hirsch MA, Iyer SS, Sanjak M. Exercise-induced neuroplasticity in human Parkinson's disease: What is the evidence telling us? Parkinsonism Relat Disord. 2016;22(Suppl 1):S78-81.
36. Hirsch MA, Farkey BG. Exercise and neuroplasticity in persons living with Parkinson's disease. Eur J Phys Rehabil Med. 2009;45(2):215-29.

37. Rochester L, Baker K, Hetherington V, Jones D, Willems AM, Kwakkel G, et al. Evidence for motor learning in Parkinson's disease: Acquisition, automaticity and retention of cued gait performance after training with external rhythmical cues. Brain Res. 2010;1319:103-11.

Received in 12/03/2015 Recebido em $03 / 12 / 2015$

Approved in 03/08/2017 Aprovado em 08/03/2017 